\title{
Viruses and athletes
}

\author{
C R Madeley
}

Infections with viruses are common, so common that the comment by the doctor "It's probably a virus" excites little surprise and less anxiety in most patients. The implications are that the patient will recover fully without significant after effects and that there will be no specific treatment. Nevertheless this is a simplistic view-there are serious, and even lethal, virus infections and there are an increasing number of antiviral drugs for use in specific circumstances. HIV (the cause of AIDS), Ebola, and rabies viruses are examples of viruses that can kill, with wide variations in the speed with which they do so, but the vast majority of common (often upper respiratory) infections are more of a nuisance than anything more sinister to the individual, although, collectively, they have economic importance to the community in lost work days. The perceived triviality of many virus infections means that confirmation of the involvement of a virus and its identification are rarely attempted except in hospital, and "It's probably a virus" usually terminates the investigation. To a professional virologist, this is both understandable and frustrating - we can identify viruses as infecting agents, and would like to do so on every occasion but it is too difficult and usually too expensive outside of the hospital context.

For athletes, viruses are important in two ways: firstly, as agents that may easily be spread among them, during competitive games in particular. In contact sports such as wrestling and rugby, skin infections such as herpes may be spread very readily ("herpes gladiatorum"), but other viruses may be transmitted by droplets during team talks or other forms of association. Secondly, viruses may be important to individual athletes who are training to a high level by interfering with training during the acute stage of an illness and, in some, by leaving long term sequelae. Here there is a wide belief that viruses are directly involved, but hard data to underpin this belief are scanty and must be augmented if the aim is prevention.

Viruses differ in the body systems they infect and the extent of the damage they do. It is not sufficient just to confirm that a virus is involved; we need to know which one(s) if we want to do anything about it/them. Vaccines for viruses work well, and it is comparatively easy to develop an effective one to a virus that spreads systemically throughout the body during pathogenesis.
Whether an individual, athlete or not, is infected, or affected, by an infectious agent will be influenced by his/her immune status. Training, overtraining, and over-reaching ${ }^{1}$ will alter the parameters of immune function that we can measure and is therefore likely to affect the outcome, in the short term with respect to how quickly the agent is eliminated, and in the long term with respect to how quickly previous levels of fitness and performance are regained. This recovery will also be influenced by any psychological effects of the episode. For example, post-influenza depression ${ }^{2}$ is not uncommon. Damage and recovery after a virus infection is a complex of interacting factors and any attempt to analyse and understand them will require a well planned and comprehensive prospective study. Is it important enough to put time, effort, and other resources into finding out? This question will be returned to later after the requirements to satisfy a virologist are outlined and a discussion of the issues from a virologist's point of view. Firstly, what does virus diagnosis and surveillance require?

\section{Diagnosing a viral infection}

These are the methods used for identifying specific virus activity, both during acute illness and afterwards. Diagnosis at the acute stage is essential if any long term effects are to be established.

METHODS

This is treated in more detail by Halonen and Madeley. ${ }^{3}$ Briefly, there are two arms to diagnosis. The first is isolating/identifying the virus and the second is monitoring the body's response to it, either by showing the appearance of specific antibodies in serum (and, increasingly, in saliva) or a lymphocyte response. The latter is confined in virology almost entirely to research laboratories, but documenting an antibody response (and, to a lesser extent, virus isolation/identification) is widely available in diagnostic virology laboratories and many microbiology ones.

Looking for an antibody reaction (serology) has significant disadvantages in the present context because it only shows what has happened after the event, and does not define it accurately in time. To show whether a virus has affected an athlete's performance, it is necessary to pinpoint to the nearest day when the episode began. Serology is not precise enough for this. Moreover, it provides no information 


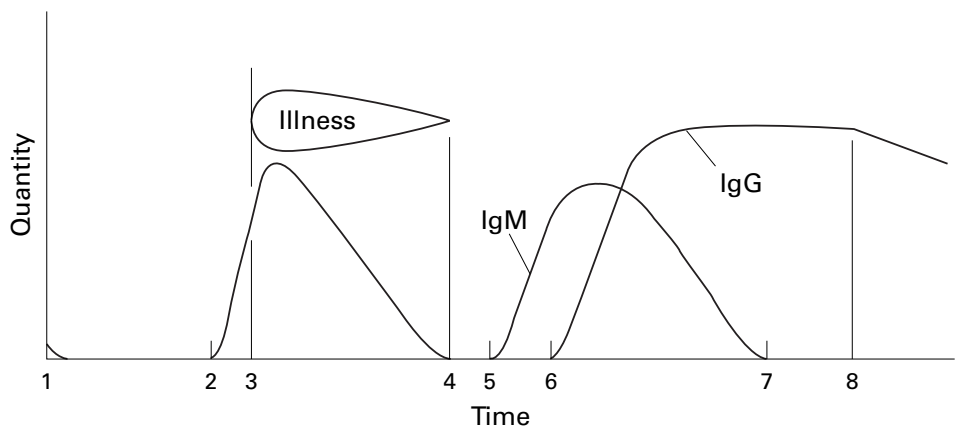

Figure 1 Schematic graph of a typical acute virus infection. The $x$ axis represents time but the numbers are events (1-8). The y axis represents quantity. The cycle begins with exposure to the virus (point 1), at which time a small amount of virus is theoretically detectable. Between points 1 and 3 is the incubation period, ending with the onset of illness. Shortly before this, virus will reappear (point 2) but is rarely detected unless specifically sought. The curve of virus detectability rises to a peak as the patient develops symptoms and will, in most cases, decline to undetectable levels (point 4) after about five days. This period of detectability will vary considerably depending on the virus and the overall immune competence of the victim. It may persist for a considerable time and, in those who are seriously compromised, possibly for life. From about one week after the start of the illness (point 5), serum antibody becomes detectable, first IgM class, followed by long term IgG (point 6). IgM antibody declines over about one month (to point 7) while IgG, after reaching a high level, will decline very slowly (from point 8) and may remain detectable for the rest of the patient's life. Individuals will show variations in this pattern but routine diagnosis is based on the assumption that the deviations are relatively minor. They usually are. Reprinted with permission from Halonen and Madeley. ${ }^{3}$

Table 1 Techniques commonly used to detect virus or viral components (NB no single technique is applicable to all viruses)

\begin{tabular}{llll}
\hline Method & Target & $\begin{array}{l}\text { Time taken from } \\
\text { receipt of specimen } \\
\text { to diagnosis }\end{array}$ & $\begin{array}{l}\text { Fragility of } \\
\text { specimen }\end{array}$ \\
\hline Isolation & Infectious virus & 2 days-3 weeks & High \\
Immunofluorescence & Infected cells & $2-3$ hours & Moderate \\
Enzyme immunoassays & Viral antigen & $2-3$ hours & Low \\
Electron microscopy & Complete virus & $15-30$ minutes & Low \\
Genome amplification $\ddagger$ & Viral nucleic acid & Overnight & Moderate \\
\hline
\end{tabular}

${ }^{\star}$ Rate of decay with time during transit to the laboratory.

†Dependent on the amount of virus in the specimen and whether the virus grows quickly or slowly. $\ddagger$ By polymerase chain reaction (PCR), nucleic acid sequence-based amplification (NASBA), ligase chain reaction (LCR), etc.

on which organs were involved. The effect on the respiratory system, for example, will not be the same as on the musculoskeletal one. Lastly, for technical reasons, serological tests are not available for every virus. Some viruses lack a group antigen and have too many serotypes for serology to be possible-for example, rhinovirus, the cause of the common cold, has over 100 serotypes. It would be impractical to test each serum over 100 times to get an answer. Although serological examination is cheap compared with isolation/identification, carrying out a full screen on all possible viruses would be a major undertaking in one individual and would be quite impractical in a study group large enough to draw useful statistical conclusions.

The alternative of looking directly for the virus (isolation or identification) has a number of advantages despite the greater cost. The first is that it links any associated illness to a narrow window in time, and the diagnosis can be made at the acute stage. This is illustrated in fig 1, which shows the profile of a typical acute infection with normal recovery. Virus is present at detectable levels during the early stage of the illness (usually for the first five days or so) while serum antibody does not appear until about a week after onset.
Table 1 lists the techniques commonly used to find virus or virus components at the acute stage. Isolation of virus in cell culture and electron microscopic examination of stool specimens have the advantages of being "catch-all" techniques, which require no prior decisions on which viruses are being sought. A suitable cell type must be available for isolation or there must be enough virus present for microscopy.

Other methods require specific reagents and will detect a narrower range of viruses, often only a single type. They must therefore be repeated to search for a variety of viruses, thereby adding to the cost. Newer techniques such as the polymerase chain reaction (PCR) and other forms of nucleic acid (genome) amplification have the advantage of great sensitivity. This extra sensitivity may be useful in the follow up of acute infections (see below).

In neither serology nor isolation/ identification is a single technique suitable for all viruses, and a combination is needed. Monitoring the infections of a group of athletes increases the work exponentially as more subjects are recruited and the net is cast wider. Cell culture is expensive to provide and labour intensive to use. Cells must be grown in defined medium and maintained in it for up to three weeks while small amounts of virus grow to detectable levels. Each culture has to be examined under a microscope every two to three days to see the effects of virus growth on the cells. Any putative viruses (and other material in the specimen that may be toxic for the cells in culture and can mimic virus induced degeneration closely) must then be identified by further tests, such as neutralisation and immunofluorescence. Not surprisingly, diagnosis by isolation, which to be adequately comprehensive requires inoculation of up to three different cell types, is expensive and, because the cultures have to be kept and regularly examined for longer, negative specimens are paradoxically more expensive than positive ones. Not only that, a negative result does not prove that no virus was present in the specimen, only that no virus grew. Hence, if the clinical picture is very "virus-like", it may be necessary to consider repeating the attempt at isolation from the original specimen.

\section{SUITABLE SPECIMENS}

It is trite but true that virus diagnostic methods are only as good as the specimens on which they are used. For isolation/identification, the specimen must contain sufficient virus or viral components for them to be found and this raises two important aspects. The first is that it is easier to take a bad specimen than a good one-taking a good one is usually more uncomfortable for the patient. The second is that, to prove that a virus is infecting, and therefore likely to be affecting, a specific organ, it is more convincing to take the specimen from that organ. Unfortunately, not all parts of the body are readily accessible for specimen taking. While specimens may be taken easily from the skin - for example, herpes simplex from cold sores-or with a little more difficulty from the nasopharynx-for example, 
Table 2 Viruses primarily infecting man $^{1}$

\begin{tabular}{|c|c|c|c|c|}
\hline Virus family & Members & $\begin{array}{l}\text { No of } \\
\text { serotypes }\end{array}$ & $\begin{array}{l}\text { Lipid outer } \\
\text { membrane }\end{array}$ & Stability \\
\hline \multicolumn{5}{|l|}{ DNA viruses } \\
\hline \multirow[t]{4}{*}{ Herpesviruses } & Herpes simplex ${ }^{\star}$ & 2 & Yes & Low \\
\hline & Varicella zoster $\star$ & 1 & Yes & Low \\
\hline & Cytomegalovirus & 1 & Yes & Low \\
\hline & Epstein Barr virus * & 1 & Yes & Low \\
\hline Adenoviruses & & 47 & No & Moderate \\
\hline \multirow{3}{*}{ Paporaviruses } & Wart* & $>60^{2}$ & No & High \\
\hline & BK & 1 & No & High? \\
\hline & JC & 1 & No & High? \\
\hline Hepatitis $B^{\star}$ & & Mutants $^{3}$ & No & High \\
\hline Parvoviruses & Adenovirus-associated & $?$ & No & $?$ \\
\hline \multirow{5}{*}{ Poxviruses } & Vaccinia ${ }^{\star}$ & 1 & No & High \\
\hline & Cowpox & 1 & No & High \\
\hline & Molluscum contagiosum ${ }^{\star}$ & 1 & No & High \\
\hline & Orf & 1 & No & High \\
\hline & Pseudocowpox & 1 & No & High \\
\hline \multicolumn{5}{|l|}{ RNA viruses } \\
\hline Reoviruses & & 3 & No & Moderate \\
\hline Orthomyxoviruses ${ }^{\star}$ & Influenza A & Many $^{4}$ & Yes & Low \\
\hline \multirow[t]{4}{*}{ Paramyxoviruses } & Influenza B & Several $^{5}$ & Yes & Low \\
\hline & Parainfluenza* & 4 & Yes & Low \\
\hline & Measles & 1 & Yes & Low \\
\hline & Respiratory syncytial virus & $1^{6}$ & Yes & Low \\
\hline Rotavirus & & At least 5 & No & High \\
\hline \multirow[t]{6}{*}{ Picornaviruses } & Poliovirus & 3 & No & High \\
\hline & Coxsackie A & 23 & No & High \\
\hline & Coxsackie $B^{\star}$ & 6 & No & High \\
\hline & Echovirus & 31 & No & High \\
\hline & Enterovirus & 4 & No & High \\
\hline & Rhinovirus & $>100$ & No & Moderate \\
\hline Caliciviruses & & 5 & No & High \\
\hline Small round structured viruses & & $>5$ & No & High \\
\hline \multirow[t]{2}{*}{ Retroviruses } & $\mathrm{HIV}^{\star}$ & any $^{7}$ & Yes & Low \\
\hline & HTLV & 2 & Yes & Low \\
\hline Hepatitis A & & 1 & No & High \\
\hline Hepatitis $C^{\star}$ & & $\sim 5$ & Yes & Low \\
\hline Togaviruses & Rubella & 1 & Yes & Low \\
\hline
\end{tabular}

${ }^{1}$ Add to this list $>200$ viruses transmitted from animals to man by biting insects, those transmitted to man directly from animals and many others confined to the tropics.

${ }^{2}$ Genotypes rather than serotypes.

${ }^{3}$ One basic serotype but an increasing variety of mutants.

${ }^{4}$ Appear seqentially - only 2-3 at any one time.

${ }^{5}$ Similar to influenza A but only one present at a time.

${ }^{6}$ Two subgroups, but very similar antigenically.

${ }^{7}$ Highly mutable but several subgroups.

*Viruses more likely to be important in a sports context.

for most upper respiratory infections-it is much more invasive to take specimens from muscle (relevant to the present discussion), the heart, or the lungs. It is not surprising that the evidence that viruses affect these organs is more scanty and indirect than for skin or the upper respiratory tract.

To grow and survive, viruses need suitable living cells - that is, cells that are respiring and metabolically active. Consequently, virus in any specimen will die off exponentially until it is put into culture, and therefore there is some urgency in getting it to the laboratory as quickly as possible. Cooling, but not freezing, it will slow the rate of decay. It is this fragility more than anything else that has inhibited virus diagnosis in general practice in the United Kingdom and elsewhere, except in rare circumstances when individual enthusiasm has overcome these obstacles. A delay in the specimen reaching the laboratory will cast doubt on the validity of a negative result, and respiratory viruses are particularly fragile.

Other components may be less fragile, but nothing in a virus is totally stable at room temperature. Viruses may be stored at $-70^{\circ} \mathrm{C}$ and below, but there are still significant losses during freezing and thawing, particularly those viruses in which the outer coat is a lipid envelope (table 2).

WHICH VIRUSES?

If all the different serotypes are included (and with some common viruses, such as rhinoviruses and enteroviruses, different serotypes are effectively different viruses in diagnostic terms), there are nearly a thousand viruses which either infect humans or can be transmitted from animals. Table 2 lists the common viruses circulating in temperate climes, such as the United Kingdom, with some of their distinguishing characteristics. A similar list for the tropics would have to be extended considerably to include insect transmitted (arbo-) viruses, such as dengue and Japanese encephalitis.

The viruses listed in table 2 are, mostly, associated with particular disease syndromes. The evidence that they cause disease is either direct (virus found in the lesions) or indirectly inferred from finding the virus regularly in other less accessible sites when the typical syndrome is present. Where viruses may be implicated as possible causes of damage to athletic performance, this may be too simple a concept. Because it is difficult to take a specimen from 


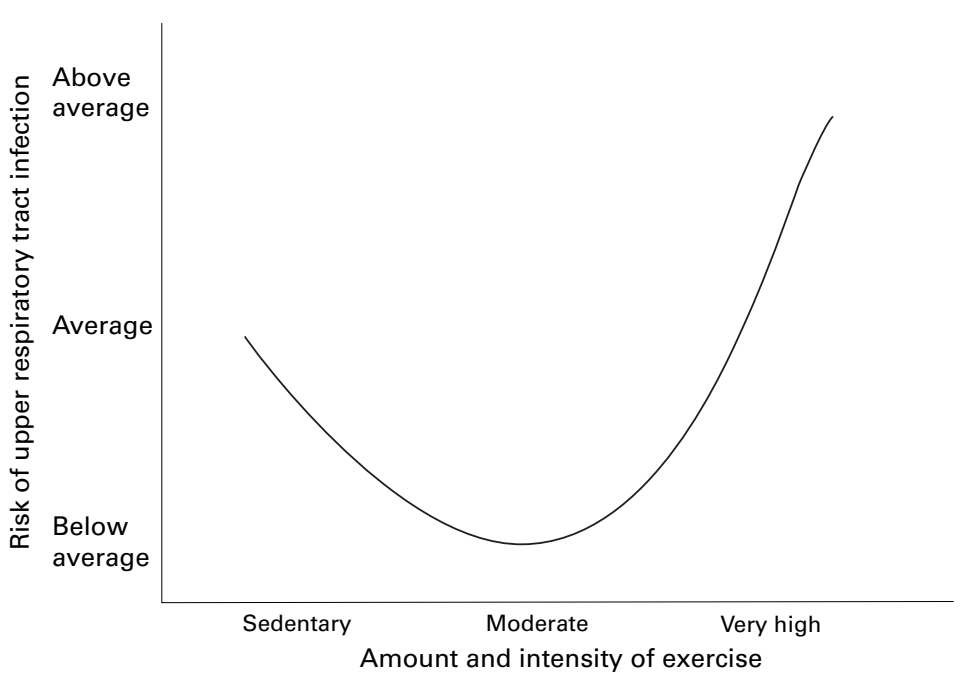

Figure 2 Graph to show the approximate relation between the amount of exercise taken by individuals and the effects on their immune systems generally, reflected in their susceptibility to respiratory tract infections. Very high exercise levels may therefore increase susceptibility. Reproduced with permission from Nieman. ${ }^{5}$

skeletal muscle, we know next to nothing about how frequently viruses invade muscle, and individual infections may be more, or less, extensive, or persist for longer, than we believe at present.

For example, a child with leukaemia died a few years ago with respiratory complications. At the postmortem examination, the only pathogen isolated from his lung tissue was a rhinovirus, one of the causes of the common cold. Rhinoviruses may be recovered from the nasopharynx in a considerable number of upper respiratory tract infections but specimens from the lower tract (lower trachea, bronchi, bronchioles, and alveoli) are ethically inaccessible in routine practice. How frequently do rhinoviruses invade the lower tract? Is it only in those who are more extensively affected? Is it inherently more likely in some individuals than others and, if so, is there anything we can measure to identify who they are? Is this relevant to infections in athletes?

OTHER AGENTS

Viruses are not the only infectious agents that may affect physical performance. Bacteria, fungi, and parasites may contribute, separately, together or sequentially. Whooping cough, aspergillus, or malaria can all have significant consequences and should not be forgotten. Bacteria may infect secondarily the tissues already breached by viruses and prolong the pathology. Nevertheless, viruses, which often cause systemic infections, are probably responsible for most of the common and more significant damage.

\section{A prospective study}

Given the difficulties of diagnosing virus infections from general practice-too difficult to carry out reliably and too expensive and too late to allow prevention or treatment-it will not be any easier from home, gymnasium, running track, or training ground. Is the information of sufficient value to justify making a special effort to collect it?
Substantial money is spent in training athletes and sending them to compete at venues all around the world. All the time, effort, and money can be wasted if a virus impairs performance or prevents competition. However, if the culprit can be identified, prevention or treatment can be considered in the future.

Attempts have been made to link viruses with longer term debility such as chronic fatigue syndrome (CFS; also known as myalgic encephalomyelitis (ME) and post-viral fatigue syndrome (PVFS)). The role of viruses in these conditions in the general population and in other underperforming athletes is presumed, but often with only circumstantial evidence to back it up.

Good evidence of viral involvement in reducing physical performance, short or long term, would have wider implications than just for athletes. What would be needed to collect scientifically valid data? It would be necessary to recruit a group of athletes who were just starting serious training towards a specific event, and following their progress, measuring their developing fitness and changes in their immunological status, identifying any infectious episodes, and measuring their effects. This would require regular weekly assessments of their performance and weekly blood specimens for estimates of immune parameters (total and differential white cell counts, response to stimuli, including counts of CD4, CD8, and NK lymphocytes), interleukin levels, and antibody titres (at least weekly specimens for virology and other microbiology with increased frequency if an infection is detected).

It is now well accepted that moderate training produces measurable improvements in immunological function, ${ }^{4}$ but overtraining can over-ride this, as illustrated in fig 2 . Within this overall pattern, individuals vary considerably, ${ }^{4}$ such that average values may not be very helpful unless large numbers are included. For this reason, it may be more realistic to study a few individuals in depth, monitoring their experience(s) in detail. This is a conclusion supported by the number of assessments outlined below. The effort and cost required to study 100 or more subjects would be large compared with any likely resources.

A compromise number of about 10 athletes is probably the upper limit for carrying out even a pilot study. They should be following a course of training towards a high level of fitness, preferably with a defined goal such as a major athletic competition on a specific date. They would have to be willing not only to carry out the training regularly and conscientiously, but also to keep records, to collect some specimens, and to allow others to be taken. As will be seen below, this is a major undertaking and a high level of both sports and scientific motivation would be needed.

The following regular assessments would be made to provide a baseline of fitness and immunological status and to identify the presence of potential pathogens before they cause problems. 
(1) Fitness. This would be assessed weekly by a sports physiologist using standard methods appropriate to the sport in question. They would probably be no different from those performed routinely but would have to be recorded on a previously prepared assessment form.

(2) Virology. Weekly nose and throat swabs would need to be taken, placed in virus transport medium, and delivered to the virus laboratory early enough for culture that day. Faecal specimens would also be needed. The residual parts of the other specimens would be stored at $-70^{\circ} \mathrm{C}$ for reanalysis if necessary.

(3) Bacteriology, mycology, and parasitology. Weekly throat swabs and faeces would be taken as necessary to monitor the presence of throat and stool pathogens.

(4) Immunology. Not less than $50 \mathrm{ml}$ heparinised whole blood would need to be taken weekly to cover all the relevant assessments of lymphocyte numbers, identity, and function, measurement of some lymphokine concentrations, provision of a sample of serum for virus antibodies, and for any possible future reassessments. The implications of this are discussed below under ethical considerations.

(5) Duration. The study would have to be over a complete training and competition season which, in the context of track and field athletics, would be a whole year. With other sports, the "season" is becoming longer and longer, but may have a number of peak periods of competition which could make definition of a virus role more difficult. Whatever the sport, the essence would be to cover the training build up and the competitions/matches that follow.

If the longer term effects, if any, of viruses (or other agents) on athletes are to be documented and understood, further follow up of any infections are necessary. This is not as easy as it appears. With the standard techniques outlined above, virus is not usually detectable in an acute infection for longer than five days. If a virus continues to influence the performance of an individual, it must be interfering with a physiological process somewhere in the body. Which process, where, and how? In what form does the virus persist, in which organs (possibly elsewhere in the body, as well as in those affected), and by what mechanism does it affect organ function? None of these questions are easy to answer.

Those patients unlucky enough to contract facial herpes know that the lesions return again and again. The recurrences are related to various factors, including sunlight, other infections, and physical and emotional stress. Between attacks, the virus lies inaccessibly dormant in the sensory root ganglia of the central nervous system, but is readily detectable in the new skin lesions of a recurrence.

In longer term disability such as CFS, it is voluntary muscle that appears to be affected. Weakness has been documented by stimulation myography, ${ }^{6}$ but this is more difficult to investigate virologically than, for example, the immediately accessible skin lesions of herpes simplex. Muscle biopsy would have to be performed to draw any valid conclusions, a procedure that few ethical committees would accept, especially if it meant taking several biopsy specimens, one in the acute phase and another about six months later. In theory, follow up biopsy samples would be taken only from those with persisting impairment, but these would not be known at the acute stage. Hence, all the subjects would have to have samples taken both at the acute stage and again later to provide normal controls. Even if, improbably, the subjects agreed to this assault, it seems very doubtful that any ethical committee would concur.

If it is not ethically acceptable to sample the affected organ, it becomes difficult to answer any of the three questions posed above, except indirectly. However, demonstration of persistence anywhere in the body would be a start. Let us consider the implications of this approach with a candidate virus.

Coxsackie B (CB) viruses are linked with muscle infections. They are strongly associated with Bornholm disease (a very painful inflammation of the intercostal muscles) and have been recovered from cardiac muscle in fatal cases of cardiomyopathy. ${ }^{7}$ These, however, are rare sequelae of a gut infection, which is itself very common, particularly in children, and which only occasionally progresses beyond the gut. $\mathrm{CB}$ viruses also cause aseptic meningitis and they may then be readily isolated from the cerebral spinal fluid. Infection of the gut is confirmed by isolating the virus from faeces but this does not prove muscle involvement.

It may be possible to increase the sensitivity of detection in faeces using nucleic acid amplification techniques and show that small amounts of virus genomic material are still being excreted after complete virus can no longer be isolated. This, however, does not take us much further and to understand how and in what form the virus is persisting would require gut biopsy, which is no more acceptable ethically. Nevertheless, examining easily acquired faeces in this way would, if they were positive, show that there was something else to investigate later. However, it would not tell us how to do it.

The genome of viruses is in the form of either DNA or RNA. CB viruses are among those with RNA, while others such as herpes use DNA. In the body, cells use DNA to store (genetic) information in the nucleus and it is therefore protected from degradation. RNA is used to transfer this information, as necessary, from the nucleus to the ribosomes to provide a template for protein production. Once it has been used, it is degraded so that the cell does not accumulate information that is not being used. Within a cell therefore RNA is essentially transient and, if the RNA of CB (or any other RNA-containing viruses) persists intracellularly, in what form does it do so? It is worth noting that one RNA virus known to persist, HIV, does so by carrying with it an enzyme, reverse transcriptase (RT), the function of which is to make a complementary DNA 
(cDNA) copy of the genome. This is integrated into the host cell genome and is then able to remain in a protected environment, probably for the rest of the patient's life. RNAcontaining viruses do not, in general, have an RT and this enzyme is not found in human cells, only in some viruses. CB viruses are not among them.

A model of possibly greater relevance is provided by hepatitis $C$ virus. It too is an RNA virus which persists and which has not been shown to possess RT. Virus activity has been shown in the liver, ${ }^{8}$ but finding it does not explain how it persists. It may have a primary focus elsewhere, where low level continuing virus production repeatedly infects new liver cells. If this occurs with hepatitis $\mathrm{C}$ virus, it would be necessary to postulate a different mechanism for $\mathrm{CB}$ viruses, which convert an acute infection, producing large amounts of virus and cellular damage, into a chronic one, causing less obvious damage and releasing less virus. Invasion of another type of cell elsewhere in the body would be one explanation. Which cells? Where? Although follow up would be highly desirable, particularly of any individuals showing persisting effects, this would not be straightforward or easy.

\section{Conclusions}

The financial and emotional investment put by an individual into training to the highest level he or she can attain is immense. To find it effectively going to waste because an infection, often thought to be trivial, damages that performance must be very galling to athletes and coaches alike. We know little about the contribution of various viruses to these disappointments and even less to longer term problems such as CFS. CFS is by no means confined to high class athletes but is very difficult to investigate retrospectively, especially as current definitions require it to be present for at least six months to qualify. ${ }^{9}$

A prospective study of highly motivated athletes would help to understand CFS in others and would therefore have wider implications. Such a study would be essential if any conclusions are to be valid, although the analysis in this review makes the problems plain. Athletes in training must be well motivated to achieve their best and they would have a personal and powerful interest in any factor that might deprive them of a medal. Peak training on the verge, or even over the edge, of overtraining will leave them vulnerable to infection in addition to the stress of reaching their peak on exactly the right day. Such highly motivated individuals would provide an appropriate study group, and there would be a high expectation that they would see it through.

Since a study like this would essentially be a "fishing expedition", it would face the same problem as any similar epidemiological study"too open ended and too uncertain" of producing significant data. Nevertheless, the possibility of collecting valuable information that would also be applicable to those with CFS should provide stronger impetus, if only to raise the possibility of a definite diagnostic test for this condition.

Without a fully planned prospective study, only anecdotal evidence will remain, backed up by occasional data from biopsies etc carried out for other reasons. If the continuing concern, reinforced by pressure groups, to understand CFS is to be satisfied, some scientifically valid studies, controlled as far as possible, are essential. Outlined above is one suggestion of how it might be tackled, but is not the only possible one. Others may have different approaches, but the need for a prospective study seems clear. Given the variety of viruses that may be involved, improving on present anecdotal convictions should be the aim. There is scope for new approaches to this diagnostic challenge.

1 Budgett R. Fatigue and underperformance in athletes: the overtraining syndrome. Br f Sports Med 1998;32:107-10.

2 Christie $\mathrm{AB}$. Acute respiratory infections. In: Infectious diseases, 4th ed. Edinburgh: Churchill Livingstone, 1987: 413-74

3 Halonen P, Madeley CR. The laboratory diagnosis of viral infections. In: Mahy BWJ, Collier L, eds. Topley $\mathbb{E}$ Wilson's microbiology and microbial infections, vol 4 virology, 9th ed. London: Arnold, 1998:947-62.

4 Shephard RJ, Shek PN. Potential impact of physical activity and sport on the immune system: a brief review. Br f Sports and sport on the imm

5 Nieman DC. Upper respiratory tract infections and exercise. Thorax 1995;50:1229-31.

6 Dickinson CJ. Chronic fatigue syndrome: aetiological aspects. Eur fं Clin Invest 1997;27:257-67.

7 Melnick JL. Enteroviruses: polioviruses, coxsackieviruses, echoviruses and newer enteroviruses. In: Fields BN, Knipe DM, Howley P, eds. Fields' virology, 3rd ed. Philadelphia: Lippincott-Raven, 1996:655-712.

8 Houghton M. Hepatitis C viruses. In: Fields BN, Knipe DM, Howley P, eds. Fields' virology, 3rd ed. Philadelphia: Lippincott-Raven, 1996:1035-8.

9 Sharpe M. Chronic fatigue syndrome. Psychiatr Clin North Am 1996;19:549-73. 